\title{
Understanding congruence in person-centred counselling practice: A trainee counsellor's perspective
}

\author{
Natri Sutanti \\ School Education, Faculty of Social Science \\ University of Nottingham \\ E-mail: ttxns45@nottingham.ac.uk
}

Received: September 22, 2020; Revised: October 28, 2020; Accepted: October 28, 2020

\begin{abstract}
Congruence is a condition in therapeutic relationship that refers to accurate matching of a person's experience with awareness. In person-centred counselling, counsellor's congruence is believed as one of helpful and significant aspects that facilitates clients' growth in counselling. However, this term is quite difficult to understand as a single condition as it interrelated to the other conditions such as empathy and unconditional positive regard. Understanding congruence theoretically and practically is intriguing as well as challenging especially for a trainee counsellor that is still learning to apply theory on practice. This paper aims to critically explore congruence from person-centred counselling theory and to demonstrate some evidences of the development of congruence in practice based on the author's counselling practice as a trainee counsellor. There are three main discussions in this paper that is to explain congruence from the theoretical point of view, to understand the relation between congruence and acceptance and to explore congruence in person-centred practice. The exploration found that trainee counsellor's cultural background including condition of worth and language barrier is one of challenges in experiencing congruence within person-centred counselling practice.
\end{abstract}

Keywords: congruence, person-centred counselling, unconditional positive regard, ontological standpoint

This is an open-access article under the CC-BY-SA license.

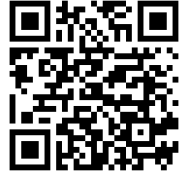

(2)

Introduction

Congruence is the third of six core conditions in person-centred counselling (PCC) theory. It is probably the most difficult concept to understand and practise (Greenberg \& Geller, 2001). Even though the concept is widely used in everyday life such as in mathematics, the meaning of congruence in the context of PCC is not as clear as its meaning in mathematics. The meaning of congruence tends to be ambiguous as an effect of the different word versions used in Rogers' theory such as genuineness, authenticity and transparency (Brodley, 2011; Cornelius-White, 2013; Tudor, 2011). Those words are commonly used interchangeably with congruence, although the meaning of each word is slightly different. Congruence refers to a broad concept of a person's openness to awareness and accurate symbolisation of experience (Rogers, 1959). It means that a 
person who is congruent is able to understand his or her feeling and thought accurately and whilst the same time experiencing unconditional positive regard and emphatic understanding. Meanwhile, authenticity for instance is a word specifically used to represent an individual's openness, transparency, the ability to be realistic, responsible to their life choices and fully aware on what they say or do (Joseph, 2017).

Regardless of the controversy in understanding the concept of congruence, ideally personcentred counsellors are expected to be congruent in the relationship with the clients during the counselling process. Practically, it is clearly stated in the person-centred and experiential psychotherapy scale (Elliot \& Westwell, 2011) for trainee counsellors that they have to genuinely and naturally convey their moment-to-moment experiencing with the client. In line with PCC theory, Rogers (1959) states that congruence is the most important therapeutic condition and needs to be prioritised in PCC practice. Congruence deserves to be the most prominent condition in PCC, because the meaning comprises the values of sincerity, clarity and purity, which are generally good catalysts in building a relationship as the basis of this counselling (AtzilSlonim, et al., 2018; Kolden, Wang, Austin, Chang \& Klein, 2018; Norcross \& Wampold, 2011).

Becoming consistently congruent in person-centred counselling is an obligation for a counsellor as it was defined in the third of six necessary and sufficient conditions of the therapeutic process by Rogers in 1957 and 1959 (Wyatt, 2002). However, putting this concept into practice is much more difficult than explaining it verbally. It is challenging to identify whether or not a counsellor is congruent during a counselling session, especially for a trainee counsellor who still learn to do self-evaluation and apply theory into practice. Trainee counsellors can be trapped on a counsellor mode and inner critics that could prevent them from being objective in evaluating their congruence. Therefore, this paper aims to explore congruence in person-centred practice from theoretical point of view and to provide some evidences of congruence from the author's training experience. There are three main discussions in this paper. First is further exploration of the correlation between congruence and the idea of human nature and an ontological standpoint. Second is the relation between congruence and acceptance that is including unconditional positive regard (UPR) and unconditional positive self-regard (UPSR). The last discussion is exploration of the counselling practice in relation to the congruence theory.

\section{The ideas of human nature and an ontological standpoint}

Putting the discussion of human nature and an ontological standpoint for exploring the concept of congruence into practice is beneficial and noteworthy. Firstly, ideas of human nature will give counsellors a philosophical overview of themselves and their clients as a human being. With this understanding, they can fully accept themselves and their clients with any conditions. Eventually, it will encourage congruence in the relationship, which will benefit the client's growth. Secondly, an ontological standpoint is fruitful to give an identity to the counsellors as it indicates the position in which the counsellors actually stands and grounds themselves when facing the clients. Furthermore, philosophical positioning also contributes in giving direction to the counselling and can be used as a measurement for congruence.

Indirectly, ideas of human nature will have some impact on the way counsellors perceives themselves as well as their clients. In PCC, Rogers (1957) postulates that human nature has fundamental basic characteristics, such as realistic, and thus a human can be trusted to move in a constructive and positive direction if they live in nonthreatening socio-environmental conditions. This concept implies that a human being is imperfect because even though they have the potential to be positive, they cannot control their living environments to always be supportive. Ultimately, it also means that an imperfect counselling practice is reasonable and for this reason, counsellors does not have to be free from any conflict before counselling, but they have to be good enough by focusing on developing self-awareness and self-control (Mearns, 2003). Holding on to the same understanding, person-centred counsellors will not have any assumptions of the clients and will instead focus on thinking about how they can facilitate the session to help the 
clients find their own solutions (Murphy, 2014). Furthermore, they will pay more attention on the client's direction. The synergy of good understanding on both sides will promote the counsellor's performance and the therapeutic relationship towards the client's positive growth.

Based on the philosophical concept of human nature, ultimately the ontological standpoint of every counselling approach can be vividly determined. By that, it is appropriate to call an ontological positioning as a counsellor's identity. Even though a counsellor grounds from the roots of humanistic counselling understanding, it is still possible to identify the exact type of humanistic approach used. Inconsistency between counsellor's ontological standpoint, theory and practice can be perceived as incongruence (Nye, Connell, Haake \& Barkham, 2018). For instance, from Rogers's PCC and Greenberg's emotion focus therapy counselling videos, it can be determined the differences of the theoretical standpoint between the two counselling theories. Rogers's counselling has characteristics such as non-directive, facilitative, reflective, and emphatic understanding, while Greenberg's therapy tends to have characteristics such as directive, good understanding, problem solving oriented, and technical skills. If a counsellor uses the PCC philosophical view to evaluate, he or she would say that Greenberg's attitudes are nearly incongruent with PCC's ontological standpoint because he tends to be more facilitative by directing rather than by following the client's flow. However, in fact, both of the therapists have explicitly practiced congruence to their own ontological standpoint by practicing counselling differently.

It cannot be denied that the ideas of human nature, ontological standpoint, and congruence are closely related to each other. This relation can be seen clearly from the first hypothesis by Rogers (1961), which asserts that "if I can provide a certain type of relationship, the other person will discover within himself the capacity to use that relationship for growth and change and personal development will occur" (p. 33). Therefore, a counsellor initially meets with the client to form a therapeutic relationship based on the full understanding of human nature and an ontological standpoint. The relationship then improves the quality of the six-sufficient conditions that would promote the client's positive growth. To successfully provide these constructive conditions, Rogers notes that it will be more beneficial for both the client and the counsellor if the counsellor can be more congruent in the relationship. Furthermore, to be more congruent, counsellors have to accept themselves, their clients and all circumstances that exist according to what they understand about human nature and then put it into action consistently with their ontological standpoint.

\section{The relation between congruence and acceptance}

Congruence is theoretically defined as a condition in which a counsellor is freely to be herself or himself within the relationship and can accurately symbolise her or his own experience in awareness (Rogers, 1959). This definition gives a clear picture of congruence which can be simply understood as harmony between self and experience. However, the discussion of congruence cannot be separated from the other two core conditions, as Rogers states that "...a part of the congruence must be the experience of unconditional positive regard and the experience of emphatic understanding" (p. 215). Therefore, to understand the concept of congruence, a definition of unconditional positive regard (UPR) and emphatic understanding is needed. UPR means experiencing warm acceptance of whatever the client is at that moment (Standal, 1954), whilst according to Rogers, UPR relates to the experience of unconditional positive self-regard (UPSR), which is a condition when any given experience can connect to the whole self-structure. Meanwhile, emphatic understanding is a way to communicate congruence and UPR to the client based on the deep understanding of the client's frame of reference (Merry, 1999).

In practice, congruence is more complex, as it can be experienced differently by each counsellor in therapy. This has been proved by Grafanaki (2002), who examined how congruence works in PCC practice. Her research shows that congruence is experienced by counsellors in 
various ways and there is no unitary phenomenon. This implies that experiencing congruence in practice is very subjective and personal. Experiencing congruence in practice cannot be separated from experiencing acceptance, including UPSR and UPR. When counsellors can accept the whole of themselves as a person who has strengths and weaknesses, at the same time, they are more able to unconditionally accept their clients as they are (Kivlighan, Kline, Gelso \& Hill, 2017; Kolden, Klein, Wang, \& Austin, 2011). Then, when counsellors have both forms of acceptance, they can be more aware of the dynamics of their feelings during the counselling process, so that they are more able to determine when their feeling is more congruent and less congruent during the counselling process.

The correlation between congruence and self-acceptance has been stated by Rogers (1959) in the theory of therapy, personality and interpersonal relationships. He uses the context of family life to explain the dynamics of congruence and acceptance in improving a relationship. Rogers states that in order to experience UPR, a parent needs to experience UPSR, which is the need for being congruent in the relationship with the child. He continued with the explanation, that if those conditions are fulfilled, the parent will emphatically understand the child's internal frame of reference and experience UPR for the child. This also applies to the therapeutic relationship, where the counsellor needs to accept herself to experience UPSR in the relationship in order to experience congruence. Therefore, to confirm the accuracy of congruence, a counsellor needs to experience UPR from the client and be able to convey her or his emphatic understanding to the client.

In addition, Standal's (1954) idea of psychological maturity also confirms the theoretical truth of the relation between congruence, UPR and UPSR. Standal states that when a person becomes psychologically mature, which is similar to being congruent, she or he would no longer introject values, nor deny or distort experiences as it becomes impossible to frustrate the need for self-regard. This implies that when counsellors are congruent, they would fully accept their experience and not be disturbed by the past experience. They would also experience UPSR which prevents them from becoming frustrated owing a lack of self-regard. Ultimately, when counsellors experience congruence in counselling, they will get a reciprocal acceptance from their clients which is known as UPR. Generally, Rogers' (1959) and Standal's explanations of congruence are almost the same. Therefore, it can be concluded that the relationship between congruence and acceptance can be defined in four stages. First, a counsellor gives UPSR to herself or himself. Second, the counsellor will experience UPR from her or his client. Third, the counsellor can experience congruence by being aware of the client's experience, her or his own experience and self-concept, which would make it impossible to become frustrated owing to sufficient self-regard. Finally, the counsellor would be able to communicate her or his understanding in empathic response, where the therapeutic development will grow.

\section{Experiencing congruence in practice}

Understanding the concept of congruence from the relation to acceptance has given an idea of internal process to achieve congruence mindset in the counselling. However, in the practice setting, congruence is still difficult to observe and assess. Rogers as the founder of person-centred counselling has never adequately explained the boundaries of how to communicate congruence to the clients (Frankel, Johnson \& Polak, 2016). It is not clear when counsellors should voice their personal feeling in the counselling and how much the counsellors can disclose their conditions of worth to their clients (Jolley, 2019). Frankel, Johnson and Polak state that the concept of congruence can be clarify using contexts of therapeutic relationship and general human relationship. It means that given context in explaining the experience of congruence can help to understand the concept. In this part, there will be explored the experience of congruence from the author's experience as a trainee counsellor. 
This study found that trainee counsellor's cultural background is one of challenges in experiencing congruence in therapeutic relationship. Every culture has different perspectives in responding to the client's situation that can be a barrier to experience congruence in practice (Sutanti, 2019). Counsellor's condition of worth is caused by different value, belief and language that can be a barrier for the counsellor to understand the client (Jayne, 2013; Tudor, 2013). As an instance, the limitation of the language proficiency makes counsellor difficult to understand the client thoroughly. This condition is exacerbated by a cultural shock and a tendency to comparing between cultures. To some extent, the focus of counselling shifts from tracking the client frame of reference to the personal conditions of worth. Trainee counsellors continue to deal with some internal barriers by utilising supervision (Nye, Connell, Haake \& Barkham, 2018). Supervision that is enable trainee to do self-evaluation can led to the development of the congruence mindset. This development is in line with the change of mindset, which has become more adaptive in a new cultural environment. Therefore, he understanding to accept one-self as a person who is imperfect and has some weaknesses besides some potentialities continues to grow throughout the training.

There are three stages that can be concluded from the author counselling experience. In the first stage, the domination of feeling incompetence as a trainee counsellor put some fear of making mistakes, anxiety about being offensive to the other cultures, and feelings of inferiority. Those feelings are a part of the conditions of worth which can shift the focus from the client to the counsellor (Jolley, ). As a result, it can cause some artificial responses to the client and the losing track of the client's story. From the author experience, there were many artificial responses during the first counselling practice that one of them is shown in the following example.

Client (female) :" ...I am really worried about somebody comes to me and describes some

Counsellor

extreme behaviour, he has done ... how I can truly accept him ..."

: "It sounds that you have a concern about him." (Video practice-1, 2017).

From the example, it can be seen that the counsellor only responds on a general meaning of what the client said. The counsellor did not respond to how the client was worries to be not genuine to someone. It shows that the counsellor was losing the focus on tracking the client frame of reference and therefore the response sounds formal and artificial. The domination of some negative thinking and feeling incompetent in the first stage can explain the artificial response from the counsellor. Referring to Standal (1954), in this context the counsellor condition could be considered as maladjusted in psychological maturity or in other words called as incongruence. It is the conditions where the counsellor experience is inconsistent with the client's values in order to avoid frustration at the lack of self-acceptance or UPSR (Boeree, 2016; Parkes, 2019). This then leads to the experience of incongruence in the therapeutic relationship.

The second stage is the development of awareness around the idea of human nature. The learning experience from supervision can help the trainee counsellors to feel more confident to their practice as well as to be aware of imperfection in counselling (Hoover, 2011; Stefano, D'luso, Blake, Fitzpatrick, Drapeau, \& Chamodraka, 2007). As a human being, counsellors allow to make mistakes and grow from their own mistakes. This understanding can promote unconditional positive self-regard, so that counsellors are able to accept their conditions and any imperfections. As a result, the level of acceptance to their clients increases. At this point, counsellors start to be able to distinguish the dynamics of their congruent feeling within the therapeutic process. Here, the example of the second stage: 
Client (male) : "I have a lot of regret about not giving my aunties enough care, and not spending enough time to them."

Counsellor : "It sounds like you are really care to your aunties and you want to do more than what you've done." (Video practice-6, 2017).

From the example, the counsellor's response has developed comparing to the first stage example. The counsellor can touch to the feeling of not enough in spending time to his aunties. The response also sounds natural and trying to understand the client's feeling of his aunties. However, the common challenge in this stage is to separate client's feeling and counsellor's feeling in regards to the distortion of the conditions of worth. In the familiar case or situations, counsellor should be able to differentiate that the client's conditions are not their conditions, so that they can still give an objective and genuine response to the client. According to Rogers's (1959), when counsellors have been able to accept themselves and UPSR, they are more likely to be able to show their UPR to the clients, so that the clients can feel accepted by the counsellors. Meanwhile, according to Standal's (1954) theory, this stage refers to the readjusted stage in his theory in which counsellors start to get more understanding of themselves and their situations.

The third stage is when the confidence has been built and the awareness of the dynamic feelings in counselling session has been developed. The counsellors become more sensitive to their feelings and can notice the changes on how they communicate their congruence. In this stage, strengthening the understanding of ideas of human nature and the ontological standpoint can help to maintain the UPSR dan UPR which encourage the congruent feeling in counselling. Congruence can be observed from the accepting attitude and emphatic responses. One example of the emphatic response as evidence of experiencing congruent is shown below.

Client (female) : “...I want to make sure that works in there is really good ... Try to get it kind of academic enough but also try to get personal reflections in my upcoming essays..."

feel?"

Counsellor : "It sounds that you want some balance in your essays. Is it what you

(Sutanti, 2018: p. 4-5).

This response is concise but can represent an emphatic understanding on how the client feel about her essays and what she wants to do for her essays. The counsellor can paraphrase the client meaning but still in her frame of reference. Furthermore, by confirming the understanding to the client, the counsellor also shows the potential of misunderstanding that could occur in the dialogue. Referring to Standal (1954), this stage has entered into stage of psychologically mature, where the experience and the self-concept do not distort the counsellor of being with the client in counselling.

Those provided examples in every stage are the subjective experience of congruence in practice from the author's perspectives that can be explained by Standal's theory of psychological maturity and Rogers' theory of personality. Rogers declares that congruence or genuineness is the most important condition in person-centred counselling (Rogers and Sanford, 1984). For the trainee counsellors, the learning process is still continuing, so the development of understanding about congruence is still growing. Initially the trainee counsellors need to be more understand with themselves and their feelings, so then they can feel what they do with in the counselling session. The ability to knowing and owning themselves then can lead to the confidence to be themselves in the therapeutic relationship (Joseph, 2017).

\section{Conclusion}

Congruence is a never-ending process, where the prevailing standard is being more or less congruent instead of differing it in the category of congruent and incongruent. Therefore, it is 
reasonable if a counsellor cannot become congruent every time, but the most important is to always maintain a congruent feeling during therapy. Experiencing congruence in therapy is important aspect for a counselor to promote the therapeutic relationship and the emergence of the six-sufficient conditions for the success of person-centred counselling practice. Holding on to the ideas of human nature can help counsellors to be more aware that they and their clients are part of human being who have potentials to grow as well as challenges such as some weaknesses. The understanding of human nature can help the counsellors to accept any conditions that clients bring to the counselling setting. The acceptance process is begun by the counsellors experiencing unconditional positive self-regard for themselves. In other words, the counsellors can accept their own conditions by knowing, owning and being themselves in the counselling relationship. This internal acceptance then leads to the acceptance towards their clients. The counsellor can provide safe environment that enables the clients to feel less judgmental and accepted by their counsellor or experiencing unconditional positive regard. By accepting themselves and their clients, counsellors can act more congruent in the counselling relationship that will better facilitate the clients' growth.

\section{Acknowledgment}

This paper is fully supported by Indonesian Endowment Fund for Education (LPDP).

\section{References}

Atzil-Slonim, D., Bar-Kalifa, E., Fisher, H., Peri, T., Lutz, W., Rubel, J., \& Rafaeli, E. (2018). Emotional congruence between clients and therapists and its effect on treatment outcome. Journal of Counseling Psychology, 65(1), pp. 51-64.

Boeree, C. G. (2016). Actualiæing Tendency. Retrieved from http://webspace.ship.edu/cgboer/rogers.html on 28 October 2020 at $09.00 \mathrm{am}$.

Brodley, B. T. (2011). In therapy. In K. A. Moon, M. Witty, B. Grant, \& B. Rice (Eds.), Practicing client-centered therapy. Ross on Wye: PCCS Book.

Cornelius-White, J. H. D. (2013). Congruence. In M. Cooper, M. O’Hara, P. F. Schmid, \& A. C. Bohart (Eds.), The handbook of person-centered psychotherapy and counseling. New York, NY: Palgrave.

Elliot, R. \& Westwell, G. (2011). Person-centred \& experiential psychotherapy scale: Introductory trainingversion $A$. (Unpublished).

Frankel, M., Johnson, M. M., \& Polak, R. (2016). Congruence: The social contract between a client and therapist. Person-Centered \& Experiential Psychotherapy, 15:2, pp. 156-174.

Grafanaki, S. (2002). On becoming congruent: How congruence works in person-centred counselling and practical applications for training and practice. In J. C. Watson, R. N. Goldman \& M. S. Warner (eds.), Client-centered and experiential psychotherapy in the 21st century: advances in theory, research, and practice. Ross-on-Wye: PCCS Books. pp. 278-290

Hoover, S. M. (2011). The Impact of an Experiential Training Program on Group Counsellor Development. Doctoral Dissertation, The University of British Columbia.

Jayne, K. M. (2013). Congruence, Unconditional Positive Regard, and Empathic Understanding in Child-Centered Play Therapy. Doctoral Dissertation, University of North Texas.

Jolley, H. K. (2019). I'm human too: Person-centred counsellors' lived experiences of therapist selfdisclosure. European Journal Qualitative Research in Psychotherapy, 9, pp. 12-26.

Joseph, S. (2017). Authenticity: How to be yourself \& why it matters. London: Piatkus.

Kivlighan, D. M., Jr., Kline, K., Gelso, C. J., \& Hill, C. E. (2017). Congruence and Discrepancy between Working Alliance and Real Relationship: Variance Decomposition and Response Surface Analyses. Journal of Counseling Psychology, 64(4), pp. 394-409.

Kolden, G. G., Klein, M., Wang, C., \& Austin, S. (2011). Congruence/Genuineness. Psychotherapy, 48, pp. 65-71. 
Kolden, G. G., Wang, C.-C., Austin, S. B., Chang, Y., \& Klein, M. H. (2018). Congruence/ genuineness: A meta-analysis. Psychotherapy, 55(4), pp. 424-433. https://doi.org/10.1037/pst0000162

Mearns, D. (2003). Developing person-centred counselling. 2nd edn. London: SAGE Publications.

Merry, T. (1999). Learning and being in person-centred counselling. Ross-on-Wye, Herefordshire, U. K.: PCCS Books.

Murphy, D. (2014). Psychotherapy, ontology and therapist positioning: why simplistic integrationist approaches don't work. Available at: https://personcentredpsych.wordpress.com /2014/05/14/psychotherapy-ontology-and-therapist-positioning-why-simplisticintegrationist-approaches-dont-work/ (Accessed: 7 December 2017).

Norcross, J. C., \& Wampold, B. E. (2011). Evidence-based therapy relationships: Research conclusions and clinical practices. Psychotherapy, 48(1), pp. 98-102.

Nye, A., Connell, J., Haake, R., \& Barkham, M. (2018). Person-centred experiential therapy (PCET) training within a UK NHS IAPT service: experiences of selected counsellors in the PRaCTICED trial. British Journal of Guidance \& Counselling, pp. 1-16. https://doi.org/10.1080/03069885.2018.1544608

Parkes, C. H. (2019). Finding My Voice: A Qualitative Exploration into the Perceived Impact of Person-Centred Counsellor Training upon Counsellors who were Adopted as a Baby. Master Dissertation, University of Chester.

Rogers, C. R. \& Sanford R. C. (1984). Client-centred psychotherapy. In H. I. Kaplan and B. J. Sadock (eds.), Comprehensive textbook of psychiatry. Baltimore: Williams and Wilkins, pp. 7488.

Rogers, C. R. (1957). A note on the nature of man. Journal of Counseling Psychology, 4 (3), pp.199203.

Rogers, C. R. (1959). A theory of therapy, personality, and interpersonal relationship as developed in the client-centred framework, in S. Koch (ed.) Psychology: a study of a science, Study I. Conceptual and systematic: Vol. 3 Formulation of the person and the social context. London: McGraw-Hill, pp. 184-256.

Rogers, C. R. (1961). On becoming a person: A therapist's view of psychotherapy. London: Constable \& Robinson.

Standal, S. W. (1954). The need for positive regards: A contribution to client-centred theory. (Unpublished doctoral dissertation). University of Chicago, Chicago, IL.

Stefano, J. D., D'Iuso, N., Blake, E., Fitzpatrick, M., Drapeau, M., \& Chamodraka, M. (2007). Trainees' experiences of impasses in counselling and the impact of group supervision on their resolution: A pilot study. Counselling and Psychotherapy Research: Linking research with practice, 7(1), 42-47, 10.1080/14733140601140378

Sutanti, N. (2018). Transcript and process analysis. (Submitted coursework essay). The University of Nottingham. (Unpublished).

Sutanti, N. (2019). Exploring the Challenges of the Non-Directive Attitude in Person-Centred Counselling in Indonesian Culture. KnE Social Sciences,3(17), pp. 37-50. https://doi.org/10.18502/kss.v3i17.462

Tudor, K. (2011). Rogers' therapeutic conditions: A relational conceptualization. Person-Centered \& $\begin{array}{llll}\text { Experiential Psychotherapies, 10(3), 165-180, } & \text { pp. }\end{array}$ https://doi.org/10.1080/14779757.2011.599513

Tudor, K. (2013). Person-Centered psychology and therapy, ecopsychology and ecotherapy. Person-Centered \& Experiential Psychotherapies, 12(4), pp. 315-329, https://doi.org/10.1080/14779757.2013.855137

Video practice-1. A personal video of counselling practice in a small skills group of the developing professional practice and skills module. The University of Nottingham. (Unpublished). 
Video practice-6. A personal video of counselling practice in a small skills group of the developing professional practice and skills module. The University of Nottingham. (Unpublished).

Wyatt, G. (2002). The multifaceted nature of congruence within the therapeutic relationship. In G. Wyatt (ed.) Rogers' therapeutic conditions: Evolution, theory and practice: Congruence. Ross-onWye: PCCS Books, pp. 79-95. 